\title{
THE ASYMPTOTIC MANIFOLDS OF A PERTURBED LINEAR SYSTEM OF DIFFERENTIAL EQUATIONS
}

\author{
BY \\ T. G. HALLAM( $\left.{ }^{1}\right)$ AND J. W. HEIDEL $\left({ }^{2}\right)$
}

1. Introduction. It is well known that the linear system of differential equations

$$
d y / d t=A(t) y
$$

determines the asymptotic behavior of the nonlinear system

$$
d x / d t=A(t) x+f(t, x)
$$

if $f(t, x)$ is sufficiently "small". The present paper investigates this problem further. In particular, our results are motivated by two recent studies. Brauer and Wong [1] obtained quite general results on the asymptotic relationships between the solutions of (1) and (2). In $\$ 3$ we will weaken significantly the hypotheses of some of their results. Torošelidze [5] considered the problem of perturbing the asymptotic manifold of a nonlinear scalar equation. We will extend these results to the nonlinear system (2) and consider some related questions in $\$ 4$. The main result of our paper establishes that, under certain conditions, the set of all initial positions of the solutions in a certain asymptotic manifold is an open set.

Our techniques are a combination of the well-known comparison principle and the Schauder-Tychonoff fixed point theorem. Fundamental in the application of the comparison principle is a scalar equation which we will denote by

$$
d r / d t=w(t, r) .
$$

In the above we will always assume that $A(t)$ is a real valued continuous $n \times n$ matrix defined on the interval $J=[0, \infty) ; f(t, x)$ is a real valued continuous $n$-vector defined on $J \times R^{n}$ where $R^{n}$ is Euclidean $n$-space; $w(t, r)$ is nonnegative and continuous on $J \times J$ and $w(t, r)$ is nondecreasing in $r$ for $r>0$ and fixed $t \in J$. The fundamental matrix of (1) which is the $n \times n$ identity matrix at $t=t_{0}$ will be designated by $Y(t)$. The symbol $|\cdot|$ will be used to denote any convenient vector norm.

2. Preliminary results. The following result is Theorem 1 of Brauer and Wong [1].

Received by the editors June 17, 1969.

(1) Supported by NSF under grant GP-8921.

(2) Supported by NSF under grant GP-9575.

Copyright (C) 1970, American Mathematical Society 
THEOREM 0 . Let $\Delta(t)$ be a nonsingular continuous matrix satisfying

$$
|\Delta(t) Y(t)| \leqq \alpha(t)
$$

where $\alpha(t)$ is a continuous positive function for $t \geqq t_{0} \geqq 0$. Suppose, also, that $f(t, x)$ satisfies

$$
\left|Y^{-1}(t) f(t, x)\right| \leqq w\left(t,|\Delta(t) x| \alpha^{-1}(t)\right)
$$

and that equation (3) has a positive solution which is bounded on the interval $t \geqq t_{0}$. Then, corresponding to each solution $x(t)$ of (1) with $\left|x\left(t_{0}\right)\right|$ sufficiently small, there is a constant vector $c$ such that

$$
|\Delta(t)(x(t)-Y(t) c)|=o(\alpha(t)), \quad t \rightarrow \infty .
$$

The specialization of Theorem 0 to the linear case $f(t, x)=B(t) x$ will be used in one of the following theorems. As is noted by Brauer and Wong [1, Theorem 4], in this instance $\left|x\left(t_{0}\right)\right|$ is arbitrary.

THEOREM 1. Let condition (4) be satisfied and suppose that

$$
\int^{\infty}\left|Y^{-1}(t) B(t) \Delta^{-1}(t) \alpha(t)\right| d t<\infty .
$$

Then, given any solution $z(t)$ of

$$
d z / d t=A(t) z+B(t) z
$$

there exists a constant $c$ such that the order relation

is satisfied.

$$
|\Delta(t)(z(t)-Y(t) c)|=o(\alpha(t)), \quad t \rightarrow \infty
$$

In reference to Theorem 1, we note that conditions (4) and (7) imply that

$$
\int^{\infty}\left|Y^{-1}(t) B(t) Y(t)\right| d t<\infty
$$

Under this assumption for (8), much more than the above asymptotic order relationships is known about the solutions of (8). See, for example, the paper of Ráb [4] where a complete series representation is given for the solutions of (8).

3. Asymptotic behavior of equation (2). Our main result of this section, Theorem 2 below, deals with a converse problem to that considered in Theorem 0 above. Namely, for a given solution $Y(t) c$ of (1) with sufficiently small initial condition, we will establish conditions which imply that there is a solution of (2) with the asymptotic behavior (6). Our result improves a theorem of Brauer and Wong [1] since we replace a Lipschitz condition by a more general inequality. We are able to avoid the Lipschitz condition because we use the Schauder-Tychonoff fixed point theorem rather than successive approximations. 
Theorem 2 below is closely related to a result of Onuchic [3, Theorem 2]. In fact, our hypotheses (4), (5), and (A) (below) imply Onuchic's hypothesis (H) [3]. However, we do not assume (A) in Theorem 2 and Onuchic's result considers only the special case $\Delta(t)=Y^{-1}(t), \alpha(t) \equiv 1$. On the other hand, Onuchic's result is true for all initial conditions.

First we give a lemma which will also be used in the following section. This lemma indicates a connection between one of the hypotheses of Theorem 0 and the right-hand side of the comparison equation (3).

Lemma. Suppose that $w(t, r)$ satisfies the previously mentioned conditions. Then the following are equivalent.

(A) Given any number $r_{\infty}>0$ there exists a $t_{0} \geqq 0$ and a solution $r\left(t, t_{0}, r_{0}\right)$ of the equation (3) such that $\lim _{t \rightarrow \infty} r\left(t, t_{0}, r_{0}\right)=r_{\infty}$.

(B) $\int^{\infty} w(t, \lambda) d t<\infty$ for all $\lambda$ satisfying $0 \leqq \lambda<\infty$.

Proof. First we will show that (A) $\Rightarrow$ (B). Let $\lambda \geqq 0$ be given. Select $r_{\infty}>\lambda$ and let $r\left(t, t_{0}, r_{0}\right)$ be a solution of (4) satisfying $\lim _{t \rightarrow \infty} r\left(t, t_{0}, r_{0}\right)=r_{\infty}$. Choose $t_{1}>t_{0}$ so that for $t \geqq t_{1}, r\left(t, t_{0}, r_{0}\right) \geqq \lambda$. By an integration of (4) we obtain

$$
r_{\infty}=r_{0}+\int_{t_{0}}^{\infty} w\left(s, r\left(s, t_{0}, r_{0}\right)\right) d s .
$$

Hence $\int_{t_{1}}^{\infty} w\left(s, r\left(s, t_{0}, r_{0}\right)\right) d s$ is well defined. Since $r\left(t, t_{0}, r_{0}\right) \geqq \lambda$ for $t \geqq t_{1}$, and $w(t, r)$ is nondecreasing in $r$, we obtain $\int_{t_{0}}^{\infty} w(t, \lambda) d t<\infty$.

The proof of the converse will only be outlined because it is similar to the proof of Theorem 2. Only the preceding argument of the lemma is required in the proof of Theorem 2; however, the implication (B) $\Rightarrow(\mathrm{A})$ is used in the proof of Theorem 4.

Let $\int^{\infty} w(t, \lambda) d t<\infty$ for each $\lambda, 0<\lambda<\infty$; we will show that there exists a solution $r(t)=r\left(t, t_{0}, r_{0}\right)$ satisfying $\lim _{t \rightarrow \infty} r(t)=\lambda$. Choose $t_{0}$ sufficiently large so that $\int_{t_{0}}^{\infty} w(t, \lambda) d t<\lambda$. Let $t \geqq t_{0}$ and define

$$
\mathscr{T} r(t)=\lambda-\int_{t}^{\infty} w(s, r(s)) d s .
$$

The Schauder-Tychonoff theorem (Coppel [2, p. 9]) is used to verify that $\mathscr{T}$ has a fixed point.

Let $\mathscr{F}=\left\{r: r(t)\right.$ is continuous on $\left[t_{0}, \infty\right)$ and $\left.0 \leqq r(t) \leqq \lambda\right\}$. Then it can be shown that $\mathscr{T}(\mathscr{F}) \subset \mathscr{F}$ and that $\mathscr{T}$ is continuous.

The function $z(t)=\mathscr{T} r(t)$ satisfies the differential equation $z^{\prime}=w(t, r(t))$. Therefore, the functions $\mathscr{T} r$ for $r \in \mathscr{F}$ have a uniformly bounded derivative on any finite interval, namely, $\left|(\mathscr{T} r)^{\prime}(t)\right| \leqq w(t, r(t)) \leqq w(t, \lambda)$. Thus the family $\mathscr{T} \mathscr{F}$ is equicontinuous on any finite interval.

The hypotheses of the Schauder-Tychonoff theorem are satisfied, hence $\mathscr{T}$ has a fixed point which is the desired solution of (4). This concludes the proof of the lemma. 
THEOREM 2. Let the hypotheses of Theorem 0 be satisfied. Given any solution $y(t)=Y(t) c$ of (1) with $|c|$ sufficiently small, there exists a solution $x(t)$ of (2) such that

$$
|\Delta(t)(x(t)-y(t))|=o(\alpha(t)), \quad t \rightarrow \infty .
$$

Proof. Let $r(t)$ be a bounded solution of (3) and set $r_{\infty}=\lim _{t \rightarrow \infty} r(t)$. Let $c$ satisfy $|c|<r_{\infty}$, define $\eta=\left(r_{\infty}-|c|\right) / 2$ and let $T_{0}$ be sufficiently large so that

$$
\int_{T_{0}}^{\infty} w\left(t, r_{\infty}-\eta\right) d t<\eta
$$

This is possible since $\int^{\infty} w(t, \lambda) d t<\infty$ for all $\lambda$ such that $0 \leqq \lambda<r_{\infty}$ by the argument used in the first part of the proof of the lemma.

We will establish the existence of a solution of the integral equation

$$
x(t)=Y(t) c-Y(t) \int_{t}^{\infty} Y^{-1}(s) f(s, x(s)) d s, \quad t \geqq T_{0} .
$$

Consider the set

$\mathscr{F}=\left\{u: u(t)=\Delta(t) \alpha^{-1}(t) x(t)\right.$ where $x(t)$ is continuous

$$
\text { on } \left.J_{0}=\left[T_{0}, \infty\right) \text { and }|u| \leqq r_{\infty}-\eta\right\} \text {. }
$$

We define the operator $\mathscr{T}$ by

(10) $\mathscr{T} u(t)=\frac{\Delta(t) Y(t) c}{\alpha(t)}-\frac{\Delta(t) Y(t)}{\alpha(t)} \int_{t}^{\infty} Y^{-1}(s) f\left(s, \Delta^{-1}(s) \alpha(s) u(s)\right) d s, \quad t \geqq T_{0}$.

The following inequality establishes that $\mathscr{T} \mathscr{F} \subset \mathscr{F}$.

$$
\begin{aligned}
|\mathscr{T} u(t)| & \leqq|c|+\int_{t}^{\infty}\left|Y^{-1}(s) f\left(s, \Delta^{-1}(s) \alpha(s) u(s)\right)\right| d s \\
& \leqq|c|+\int_{t}^{\infty} w(s,|u(s)|) d s \\
& \leqq|c|+\int_{t}^{\infty} w\left(s, r_{\infty}-\eta\right) d s \\
& \leqq|c|+\eta=r_{\infty}-\eta .
\end{aligned}
$$

Next, it will shown that $\mathscr{T}$ is continuous. Suppose that the sequence $\left\{u_{n}\right\}$ converges uniformly to $u$ on every compact subinterval of $J_{0}$ where $\left\{u_{n}\right\}, u \in \mathscr{F}$. Let $\varepsilon>0$ and select $T_{1} \geqq T_{0}$ sufficiently large so that

$$
\int_{T_{1}}^{\infty} w\left(s, r_{\infty}-\eta\right) d s<\frac{\varepsilon}{4}
$$

Then, from (10)

$$
\begin{aligned}
\mid \mathscr{T} u(t) & -\mathscr{T} u_{n}(t) \mid \\
\leqq & \int_{t}^{T_{1}}\left|Y^{-1}(s)\right|\left|f\left(s, \Delta^{-1}(s) \alpha(s) u(s)\right)-f\left(s, \Delta^{-1}(s) \alpha(s) u_{n}(s)\right)\right| d s \\
& \quad+\int_{T_{1}}^{\infty}\left[\left|Y^{-1}(s) f\left(s, \Delta^{-1}(s) \alpha(s) u(s)\right)\right|+\left|Y^{-1}(s) f\left(s, \Delta^{-1}(s) \alpha(s) u_{n}(s)\right)\right|\right] d s .
\end{aligned}
$$


Now, the second integral on the right side of (12) satisfies

$$
\begin{aligned}
\int_{T_{1}}^{\infty}\left[\left|Y^{-1}(s) f\left(s, \Delta^{-1}(s) \alpha(s) u(s)\right)\right|+\mid Y^{-1}(s)\right. & \left.f\left(s, \Delta^{-1}(s) \alpha(s) u_{n}(s)\right) \mid\right] d s \\
& \leqq \int_{T_{1}}^{\infty}\left[w(s,|u(s)|)+w\left(s,\left|u_{n}(s)\right|\right)\right] d s \\
& \leqq 2 \int_{T_{1}}^{\infty} w\left(s, r_{\infty}-\eta\right) d s<\frac{\varepsilon}{2}
\end{aligned}
$$

By the uniform convergence there is an $N=N\left(\varepsilon, T_{1}\right)$ such that if $n \geqq N$ then

$$
\left|f\left(t, \Delta^{-1}(t) \alpha(t) u(t)\right)-f\left(t, \Delta^{-1}(t) \alpha(t) u_{n}(t)\right)\right|<\varepsilon / 2 M\left(T_{1}-T_{0}\right), \quad t \in\left[T_{0}, T_{1}\right]
$$

where $M=\sup _{\left[T_{0}, T_{1}\right]}\left|Y^{-1}(t)\right|$. Combining (12), (13), and (14) yields

$$
\left|\mathscr{T} u(t)-\mathscr{T} u_{n}(t)\right|<\varepsilon
$$

for $T_{0} \leqq t \leqq T_{1}$ and $n \geqq N$ which shows that $\mathscr{T} u_{n}$ converges uniformly to $\mathscr{T} u$ on compact subintervals of $J_{0}$. Hence $\mathscr{T}$ is continuous.

Since $\mathscr{T} \mathscr{F} \subset \mathscr{F}$ it is clear that the functions $\mathscr{T} \mathscr{F}$ are uniformly bounded. We will show that they are equicontinuous at each point of $J_{0}$. Now, for each $u \in \mathscr{F}$, the function $z(t)=\Delta^{-1}(t) \alpha(t) \mathscr{T} u(t)$ is a solution of the linear differential equation

$$
v^{\prime}=A(t) v+f\left(t, \Delta^{-1}(t) \alpha(t) u(t)\right)
$$

Since,

$$
|z(t)| \leqq\left|\Delta^{-1}(t) \alpha(t)\right||\mathscr{T} u(t)| \leqq\left|\Delta^{-1}(t) \alpha(t)\right|\left|r_{\infty}-\eta\right|
$$

and $\left|f\left(t, \Delta^{-1}(t) \alpha(t) u(t)\right)\right|$ is uniformly bounded for $u(t) \in \mathscr{F}$ on any finite $t$ interval, we see that $z^{\prime}$ is uniformly bounded on any finite interval. Therefore, the set of all such $z$ is equicontinuous on any finite interval. To see that the functions in $\mathscr{T} \mathscr{F}$ are equicontinuous at every point in $J_{0}$, consider

$$
\begin{aligned}
\left|\mathscr{T} u\left(t_{1}\right)-\mathscr{T} u\left(t_{2}\right)\right| \\
\quad=\left|\Delta\left(t_{1}\right) \alpha^{-1}\left(t_{1}\right) z\left(t_{1}\right)-\Delta\left(t_{2}\right) \alpha^{-1}\left(t_{2}\right) z\left(t_{2}\right)\right| \\
\quad \leqq\left|\Delta\left(t_{1}\right) \alpha^{-1}\left(t_{1}\right)\right|\left|z\left(t_{1}\right)-z\left(t_{2}\right)\right|+\left|\Delta\left(t_{1}\right) \alpha^{-1}\left(t_{1}\right)-\Delta\left(t_{2}\right) \alpha^{-1}\left(t_{2}\right)\right| \cdot\left|z\left(t_{2}\right)\right|
\end{aligned}
$$

where $t_{1}, t_{2}$ are in some finite interval. The right side of (16) can be made small by virtue of the equicontinuity of the family $\{z(t)\}$ and the continuity of $\Delta(t) \alpha^{-1}(t)$. Thus the family $\mathscr{T} \mathscr{F}$ is equicontinuous at each point of $J_{0}$.

All of the hypotheses of the Schauder-Tychonoff theorem are satisfied. Thus there exists a $u \in \mathscr{F}$ such that $u(t)=\mathscr{T} u(t)$; that is, there exists a solution $x(t)$ of

$$
x(t)=Y(t) c-Y(t) \int_{t}^{\infty} Y^{-1}(s) f(s, x(s)) d s .
$$

Thus $x(t)$ is a solution of (2) and possesses the asymptotic behavior of (6). This proves Theorem 2 . 
REMARK. The referee has pointed out that the nonsingularity of the matrix $\Delta(t)$ is not required in the proof of Theorem 0 . However, it is essential in our proof of Theorem 2.

4. Perturbable manifolds. Theorems 0 and 2 above indicate that, under certain conditions, there is an asymptotic manifold of solutions of the perturbed differential equation (2) which is, in some sense, generated by the equation (1). This comment motivates the following definition.

Definition 1. Let $\Delta(t), \alpha(t)$ be as given in Theorem 0. The set $S=S(\Delta, \alpha)$ which consists of all solutions $x=x(t)$ of equation (2) that satisfy the order relation (6) for some constant vector $c$, will be called the asymptotic manifold of (2) generated by (1).

We will be interested in the following property of such manifolds.

Definition 2. The set $S=S(\Delta, \alpha)$ will be called perturbable if given any solution $x_{0}(t)$ of (2) in $S$ there exists a $\delta=\delta\left(t_{0}\right)>0$ such that if $x(t)$ is a solution of (2) satisfying $\left|x\left(t_{0}\right)-x_{0}\left(t_{0}\right)\right|<\delta$, then $x(t)$ is also in $S$.

Definitions 1 and 2 are apparently new. However, the main result of this section, Theorem 3 below, has been anticipated by Toroshelidze [5] for some special scalar equations.

THEOREM 3. Let equations (2) and (3) have unique solutions to the initial value problem. Also, let there exist a $k ; k \geqq\left|I_{n}\right|,\left(I_{n}\right.$ is the $n \times n$ identity matrix $)$ such that

$$
\left|Y^{-1}(t) \Delta^{-1}(t)\right| \leqq k \alpha^{-1}(t), \quad t \geqq t_{0} .
$$

Suppose that the hypotheses of Theorem 0 are satisfied and that $w(t, r) r^{-1}$ is nondecreasing in $r$ for $r>0$ and each fixed $t, t \geqq t_{0}$. If condition (A) of the lemma holds then the asymptotic manifold $S(\Delta, \alpha)$ of (2) generated by (1) is perturbable.

Proof. Let $x=x\left(t, t_{0}, x_{0}\right)$ be any solution of (2) such that $x \in S$. Then there is a solution $y(t)=Y(t) c$ of (1) such that

$$
|\Delta(t)(x(t)-y(t))|=o(\alpha(t)), \quad t \rightarrow \infty .
$$

Since (4) holds, we obtain the existence of a constant $M>0$ such that $|\Delta(t) x(t)|$ $\leqq M \alpha(t), t \geqq t_{0}$. From condition (A), corresponding to the positive number $2 M k$, there is a solution $r(t)$ of (3) which exists for $t \geqq T_{2 M}$ and $\lim _{t \rightarrow \infty} r(t)=2 M k$. Also there is a constant $T_{2 M}^{*}$ such that $\alpha(t) r(t)>k|\Delta(t) x(t)|$ for $t \geqq T_{2 M}^{*}$. Therefore, the number

$$
\varepsilon=\frac{1}{2}\left[r\left(T_{2 M}^{*}\right)-k\left|\Delta\left(T_{2 M}^{*}\right) x\left(T_{2 M}^{*}\right)\right| \alpha^{-1}\left(T_{2 M}^{*}\right)\right]
$$

is positive. By continuous dependence on initial conditions, there is a $\delta>0$ such that if $\tilde{x}\left(t, t_{0}, x_{0}\right)$ is any solution of (2) such that $\left|x_{0}-\tilde{x}_{0}\right|<\delta$, then

Therefore

$$
\left|x\left(T_{2 M}^{*}, t_{0}, x_{0}\right)-\tilde{x}\left(T_{2 M}^{*}, t_{0}, x_{0}\right)\right|<\varepsilon \alpha\left(T_{2 M}^{*}\right)|k| \Delta\left(T_{2 M}^{*}\right) \mid .
$$

$$
k\left|\Delta\left(T_{2 M}^{*}\right)\left(x\left(T_{2 M}^{*}, t_{0}, x_{0}\right)-\tilde{x}\left(T_{2 M}, t_{0}, \tilde{x}_{0}\right)\right)\right|<\varepsilon \alpha\left(T_{2 M}^{*}\right) .
$$


Since $k\left|\Delta(t) x\left(t, t_{0}, x_{0}\right)\right| \leqq r(t) \alpha(t),(17)$ and (18) imply that

$$
k\left|\Delta\left(T_{2 M}^{*}\right) \tilde{x}\left(T_{2 M}^{*}, t_{0}, x_{0}\right)\right| \leqq r\left(T_{2 M}^{*}\right) \alpha\left(T_{2 M}^{*}\right) .
$$

Since $\tilde{x}(t)=\tilde{x}\left(t, t_{0}, \tilde{x}_{0}\right)$ is a solution of the integral equation

we obtain

$$
\tilde{x}(t)=Y(t) Y^{-1}\left(T_{2 M}^{*}\right) \tilde{x}\left(T_{2 M}^{*}\right)+Y(t) \int_{T_{2 M}^{*}}^{t} Y^{-1}(s) f(s, \tilde{x}(s)) d s,
$$

$$
|\Delta(t) \tilde{x}(t)| \alpha^{-1}(t) \leqq k\left|\Delta\left(T_{2 M}^{*}\right) \tilde{x}\left(T_{2 M}^{*}\right)\right| \alpha^{-1}\left(T_{2 M}^{*}\right)+\int_{T_{2 M}^{*}}^{t} w\left(s,|\Delta(s) \tilde{x}(s)| \alpha^{-1}(s)\right) d s .
$$

The application of a well-known integral inequality (cf. Coppel [2, p. 35]) leads to

$$
|\Delta(t) \tilde{x}(t)| \alpha^{-1}(t) \leqq r(t) \leqq 2 M k, \quad t \geqq T_{2 M}^{*} .
$$

The solution $\tilde{x}(t)$ of (2) is a solution of the linear nonhomogeneous equation (8) where

$$
B(t)=f(t, \tilde{x}(t))[\Delta(t) \tilde{x}(t)]^{T} \Delta(t) /\|\Delta(t) \tilde{x}(t)\|_{e}^{2}
$$

where $\|\cdot\|_{e}$ denotes the Euclidean norm. From (20) and (5), letting $N$ be an appropriate norm constant, it follows that

$$
\begin{aligned}
\left|Y^{-1}(t) B(t) \Delta^{-1}(t) \alpha(t)\right| & \leqq N\left|Y^{-1}(t) f(t, \tilde{x}(t))\right|\left|\Delta(t) \tilde{x}(t) \alpha^{-1}(t)\right|^{-1} \\
& \leqq N w(t, 2 M k)(2 M k)^{-1}
\end{aligned}
$$

By the lemma in $\S 3, \int_{T_{2 M}^{*}}^{\infty} w(t, 2 M k) d t<\infty$ which implies that (7) holds. Therefore by Theorem 1, $\tilde{x} \in S(\Delta, \alpha)$. This completes the proof of the theorem.

We now consider the converse of this theorem, but only for a special case, namely, the scalar equation (3) and the corresponding linear equation

$$
d r / d t=0 .
$$

The following result is also of interest because it gives information about the class of equations (3) which are used in the comparison technique.

THEOREM 4. Suppose that $w(t, r)$ satisfies the conditions of Theorem 3. Let $S(1,1)$ be the positive solutions of (3) which satisfy (6) for some positive number $c$. Suppose that $S(1,1) \neq \varnothing$. Then $S(1,1)$ is perturbable if and only if condition (A) is satisfied.

Proof. By Theorem 3, condition (A) is sufficient for $S(1,1)$ to be perturbable. Now suppose that $S(1,1)$ is perturbable. If all of the positive solutions of $(3)$ are bounded then condition (B) is clearly satisfied and hence also condition (A) by the lemma. Suppose that (3) has both positive bounded and positive unbounded solutions. Then there is a $t_{1} \geqq t_{0}$ and two positive solutions $r_{\beta}=r\left(t, t_{0}, \beta\right)$ and $r_{\gamma}=r\left(t, t_{0}, \gamma\right)$ such that $r_{\beta}$ is a bounded solution of (3) and $r_{\gamma}$ is an unbounded solution of (3). Note that $r_{\gamma}$ need not exist for all time $t \geqq t_{1}$. Let $\beta=\sup \beta$ where the supremum is taken over all initial positions $\beta$ such that $r_{\beta}\left(t, t_{1}, \beta\right)$ is positive and bounded. Also let $\tilde{\gamma}=\inf \gamma$ where the infinum is taken over all initial conditions $\gamma$ such that $r_{y}\left(t, t_{1}, \gamma\right)$ is positive and unbounded. It is clear that $\tilde{\beta}=\tilde{\gamma}$. 
It is clear that the solution $\psi(t)=\psi\left(t, t_{1}, \tilde{\beta}\right)=\psi\left(t, t_{1}, \tilde{\gamma}\right)$ separates the first quadrant into sectors where solutions below $\psi(t)$ are bounded and solutions above $\psi(t)$ are unbounded. We will now show that $\psi(t)$ is unbounded. If $\psi(t)$ is bounded, then $\psi(t) \in S(1,1)$. Since $S(1,1)$ is assumed perturbable, there is a $\delta>0$ such that if $r$ is any solution of (3) that is initially within $\delta$ of $\psi(t)$, then $r$ is also in $S(1,1)$. This is a contradiction to the definition of $\psi(t)$, and thus $\psi(t)$ is unbounded.

Let the right maximal interval of existence of $\psi(t)$ be $\left[t_{1}, \sigma^{+}\right)$. Suppose $\sigma^{+}=\infty$ and let $\lambda>0$. Then, by continuous dependence on initial conditions and the fact that $\lim _{t \rightarrow \infty} \psi(t)=\infty$, there is a bounded positive solution $r(t)$ of (3) defined on $\left[t_{1}, \infty\right)$ and satisfying $\lim _{t \rightarrow \infty} r(t) \geqq \lambda$. Therefore $\int_{T}^{\infty} w(t, \lambda) d t<\infty$. The lemma now shows that condition (A) holds.

In the case $\sigma^{+}<\infty$, a similar argument establishes the existence of the bounded solution $r(t)$ of (3) with $\lim _{t \rightarrow \infty} r(t) \geqq \lambda$.

Finally, we will give a result concerned with the perturbation of the set $R$ of all solutions $y(t)$ of (1) for which there exists a solution $x(t)$ of (2) satisfying the asymptotic relation (6)

$$
|\Delta(t)(x(t)-y(t))|=o(\alpha(t)) \text { as } t \rightarrow \infty .
$$

In the theorem below we will use the following condition which is slightly stronger than condition (A) above.

(A') Given any sufficiently large $T_{0}$ and positive constant $r_{\infty}$, there exists a solution $r(t)$ of the scalar equation (3) which is valid for $t \geqq T_{0}$ and satisfies $\lim _{t \rightarrow \infty} r(t)=r_{\infty}$.

THEOREM 5. Let the hypotheses of Theorem 2 be satisfied. In addition, suppose $\left(\mathrm{A}^{\prime}\right)$ is valid. Then the manifold $R$ is perturbable.

Proof. Let $y(t)=Y(t) c$ be a given solution in the manifold $R$. Then corresponding to an arbitrarily large $T_{0}$ and the constant $r_{\infty}=2\left|Y\left(T_{0}\right)\right||c|$, there exists a solution $r(t)$ of (3) defined on $\left[T_{0}, \infty\right)$ and satisfying $\lim _{t \rightarrow \infty} r(t)=r_{\infty}$. The proof of Theorem 2 shows that if $T_{0}$ is sufficiently large, then any solution $\tilde{y}(t)$ of (1) such that $\left|\tilde{y}\left(T_{0}\right)\right|<r_{\infty}$ is in $R$.

Let $|c-\tilde{c}|<|c| / 2$. We will show that $\tilde{y}(t)=Y(t) \tilde{c}$ is in $R$. Note that

$$
|y(t)-\tilde{y}(t)| \leqq|Y(t)||c-\tilde{c}|<|Y(t)||c| / 2, \quad t \geqq t_{0} .
$$

Hence,

At $t=T_{0}$,

$$
|\tilde{y}(t)| \leqq|y(t)|+|Y(t)||c| / 2, \quad t \geqq t_{0}
$$

$$
\left|\tilde{y}\left(T_{0}\right)\right| \leqq\left|y\left(T_{0}\right)\right|+\left|Y\left(T_{0}\right)\right||c| / 2 \leqq\left|Y\left(T_{0}\right)\right| \frac{3}{2}|c|<r_{\infty} .
$$

Therefore $\tilde{y}$ is in $R$ and $R$ is perturbable.

COROLlaRY. Under the hypotheses of Theorem 5, the conclusion of Theorem 2 holds for all initial conditions $c \in R^{n}$ where $R^{n}$ is Euclidean $n$-space. 
Proof. By Theorem 2, $Y(t) c \in R$ if $|c|$ is sufficiently small. By the proof of Theorem 5 , if $Y(t) c \in R$ and $\tilde{c}$ is such that $|c-\tilde{c}|<|c| / 2$, then $Y(t) \tilde{c} \in R$. Therefore $Y(t) c \in R$ for all $c \in R^{n}$.

REMARK. Since condition $\left(\mathrm{A}^{\prime}\right)$ is weaker than the assumption that all solutions of (3) are bounded, the above corollary further improves the corresponding part of Theorem 4 of Brauer and Wong [1]. The above corollary is consistent with the result of Onuchic [3, Theorem 2] mentioned at the beginning of $\S 3$.

Added in proof. Theorem 0 and Theorem 2 of this paper follow as corollaries of Theorem 2.9.1 in the book Differential and Integral Inequalities, Academic Press, New York, 1969, by V. Lakshmikantham and S. Leela. The authors wish to thank G. Ladas and V. Lakshmikantham for pointing out several errors in the original manuscript.

\section{REFERENCES}

1. Fred Brauer and J. S. W. Wong, On asyptotic behavior of perturbed linear systems, J. Differential Equations 6 (1969), 142-153.

2. W. A. Coppel, Stability and asymptotic behavior of differential equations, Heath, Boston, Mass., 1965. MR 32 \#7875.

3. Nelson Onuchic, Nonlinear perturbation of a linear system of ordinary differential equations, Michigan Math. J. 11 (1964), 237-242. MR 29 \#4964.

4. M. Ráb, Note sur les formules asymptotiques pour les solutions d'un système d'équations différentielles linéaires, Czechoslovak Math. J. 16 (91) (1966), 127-129. MR 32 \#5965.

5. I. A. Torošelidze, The asymptotic behavior of solutions of certain nonlinear differential equations, Differencial'nye Uravnenija 3 (1967), 926-940. (Russian) MR 36 \#2904.

FLoRida STATE UNIVERSity,

TAllahassee, Florida 32306

UNIVERSITY OF TENNESSEE,

KNOXVILLE, TeNNeSSEe 37916 